\title{
CARACTERÍSTICAS CLÍNICAS E DESFECHOS DA INFECÇÃO POR TUBERCULOSE EM RECEPTORES DE TRANSPLANTE RENAL
}

\section{Clinical characteristics and outcomes of tuberculosis infection in kidney transplant recipients}

\author{
Sávio de Oliveira Brilhante ${ }^{1}$, Silvana Daher Costa ${ }^{2}$, Guilherme Aguiar Forte ${ }^{1}$, Marina Santos Carvalho', \\ Elizabeth De Francesco Daher', Tainá Veras de Sandes-Freitas ${ }^{1,2}$, Ronaldo de Matos Esmeraldo ${ }^{2}$
}

\section{RESUMO}

Objetivo: Esse estudo objetivou avaliar as características demográficas, clínicas e os desfechos da infecção por tuberculose (TB) após o transplante renal (TxR). Métodos: Estudo de coorte retrospectiva, incluindo receptores de TxR de um único centro de transplantes localizado no Nordeste do País. Foram considerados para análise os pacientes com diagnósticos realizados no período de 2004 a $2018(n=33)$. Resultados: Os pacientes da coorte eram predominantemente homens (54,5\%), adultos jovens (39,8 $\pm 12,3$ anos), com mediana de 29,8 meses de transplante. Nove fizeram tratamento para TB latente (LTBI). O regime imunossupressor principal foi indução com Thymoglobuline $(48,4 \%)$ em associação com tacrolimo e ácido micofenólico (MPA) $(72,7 \%)$. O sintoma inicial mais frequente foi febre $(63,6 \%)$, seguido da perda ponderal $(21,2 \%)$ e tosse $(21,2 \%)$. Vinte e quatro pacientes tiveram TB localizada, cinco tiveram formas disseminadas e quatro pacientes receberam tratamento empírico sem definição de sítio. Todos foram submetidos ao tratamento com rifampicina, isoniazida e pirazinamida, com ou sem etambutol. O tempo médio de tratamento foi de $8,4 \pm 3,8$ meses. Sete pacientes apresentaram hepatotoxicidade e dois apresentaram neuropatia periférica. A concentração de tacrolimo reduziu de $5,4 \pm 1,9 \mathrm{ng} / \mathrm{mL}$ pré-tratamento para 1,6 $\pm 0,6 \mathrm{ng} / \mathrm{mL}$ pós-tratamento (menor concentração), sendo a dose aumentada em $75,5 \%$ durante o tratamento. Doze pacientes apresentaram suspensão parcial ou completa da imunossupressão durante o tratamento. $79 \%$ dos pacientes evoluíram com lesão renal aguda (LRA), sendo, de acordo com o estadiamento KDIGO, 15 pacientes eram KDIGO 1, 8 eram KDIGO 2 e 3 eram KDIGO 3, e a principal causa foi LRA pré-renal. Três pacientes morreram no curso da doença ou do tratamento. Conclusões: A TB aconteceu tardiamente no curso do transplante e febre foi o principal sintoma inicial. Houve um elevado percentual de formas extrapulmonares. Como reportado em estudos prévios, o tratamento empírico é uma realidade no contexto do transplante. O tratamento associou-se à redução da concentração do inibidor de calcineurina por provável interação com a rifampicina. Foi elevado o percentual de disfunção aguda do enxerto renal.

Descritores: Transplante Renal, Tuberculose, Imunossupressão.

\footnotetext{
Instituição:

${ }^{1}$ Departamento de Medicina Clínica, Universidade Federal do Ceará, Fortaleza/CE, Brasil

${ }^{2}$ Hospital Geral de Fortaleza, Fortaleza/CE, Brasil
}

\section{Correspondência:}

Sávio de Oliveira Brilhante

Avenida Santos Dumont, 6944, apt 401-B, CEP 60150-161 -

Fortaleza/CE, Brasil

Cel.: +55 85999140955

E-mail: saviobrilhante2009@hotmail.com

Recebido em: 02/07/2018

Aceito em: 23/08/2018

\section{INTRODUÇÃO}

$O$ risco de tuberculose (TB) em pacientes submetidos a transplante renal (TxR) é substancialmente aumentado quando comparado ao da população em geral, sendo adquirida principalmente por reativação de infecção latente ou por aquisição direta na comunidade., ${ }^{1,2}$

O uso contínuo de fármacos imunossupressores afeta a resposta imune celular, essencial na resposta imune à infecção por TB. ${ }^{3}$ Essa terapia também contribui para a ocorrência de quadros clínicos atípicos, tornando o diagnóstico um desafio.

Igualmente desafiador é o tratamento da TB em pacientes transplantados renais, em virtude principalmente do comprometimento da função renal e 
necessidade de ajuste das doses dos tuberculostáticos e da interação farmacocinética destes com os fármacos imunossupressores. ${ }^{4}$

Apesar de o Brasil abrigar o segundo maior programa de TxR do mundo ${ }^{5}$ e de ser um país endêmico em infecções por TB, há um número restrito de séries de casos publicadas em receptores de TxR. ${ }^{1,2,6-8}$ Conhecer as experiências dos centros com o diagnóstico e manejo da TB em receptores de TxR é fundamental para aumentarmos o acervo de informações acerca dessa entidade endêmica em nosso meio e sabidamente associada a comprometimento dos desfechos do transplante. ${ }^{2}$

Assim, este estudo teve por objetivo descrever as características demográficas e clínicas e os desfechos da infecção por TB em receptores de TxR de um centro único localizado no Nordeste do Brasil.

\section{MÉTODO}

Estudo observacional de coorte retrospectiva, por meio de revisão de prontuários hospitalares, incluindo receptores de TxR de um único centro de TxR localizado no Nordeste do País, o qual realiza aproximadamente 120 transplantes renais por ano, sendo aproximadamente 95\% com doador falecido. Até 2018, 2324 TxR haviam sido realizados no serviço e 1443 estavam em seguimento ambulatorial.

Foram incluídos todos os pacientes transplantados renais em seguimento no serviço, os quais receberam tratamento com tuberculostáticos entre os anos de 2004 a 2018. Foram excluídos pacientes com grande volume de informações indisponíveis ou ilegíveis.

O estudo foi desenvolvido com base em princípios legais e éticos que envolvem a pesquisa com seres humanos, estabelecidos na Resolução 466/2012, do conselho Nacional de Saúde, do Ministério da Saúde do Brasil, e foi devidamente aprovado pelo Comitê de Ética em Pesquisas em Seres Humanos do Hospital Geral de Fortaleza, garantindo o respeito e proteção ao indivíduo, bem como o sigilo dos seus dados pessoais.

As variáveis categóricas serão descritas por meio de frequência e porcentagem e as numéricas por meio de média \pm desvio padrão.

\section{RESULTADOS}

\section{- Amostra / Demografia}

Os pacientes da coorte eram predominantemente homens (54,5\%), adultos jovens (39,8 $\pm 12,3$ anos), eutróficos (índice de massa corporal 22,2 $\pm 12,3 \mathrm{~kg} / \mathrm{m} 2$ ), pardos
$(66,7 \%)$, portadores de doença renal crônica (DRC) de etiologia indeterminada (33,3\%), com mediana de 29,8 meses de TxR ao diagnóstico, os quais receberam rins com doador falecido $(66,7 \%)$ (Tabela 1). Dezessete $(51,5 \%)$ receberam globulina anti-timócito como terapia de indução e o regime de imunossupressão inicial mais utilizado foi a combinação de um inibidor de calcineurina (ICN) e micofenolato, com esteroides (57,5\%). Doze tinham PPD $>5 \mathrm{~mm}$ antes do transplante e nove destes fizeram tratamento para TB latente (LTBI) (Tabela 2).

Tabela 1 - Demografia dos pacientes

\begin{tabular}{|c|c|}
\hline Característica & $\mathbf{N}=\mathbf{3 3}$ \\
\hline Idade (anos) & $39,8 \pm 12,3$ \\
\hline \multicolumn{2}{|l|}{ Procedência } \\
\hline Ceará & 31 (94\%) \\
\hline Outros Estados & $2(6 \%)$ \\
\hline Gênero Masculino & $18(54,5 \%)$ \\
\hline Índice de Massa Corporal (kg/m2) & $22,2 \pm 12,3$ \\
\hline \multicolumn{2}{|l|}{ Etnia } \\
\hline Pardos & $22(66,7 \%)$ \\
\hline Brancos & $8(24,2 \%)$ \\
\hline Negros & $1(3 \%)$ \\
\hline Informação Não Disponível & $2(6 \%)$ \\
\hline \multicolumn{2}{|l|}{ Etiologia da doença renal crônica } \\
\hline Glomerulonefrite Crônica & $14(30,3 \%)$ \\
\hline Indeterminada & $10(30,3 \%)$ \\
\hline Nefroesclerose Hipertensiva & $6(18,2 \%)$ \\
\hline Outros & $2(6 \%)$ \\
\hline Não Disponível & $1(3 \%)$ \\
\hline Tempo de Tx ao diagnóstico (meses) & $\begin{array}{c}42,22 \pm 44,82 \\
\text { Mediana }=29,75\end{array}$ \\
\hline \multicolumn{2}{|l|}{ Tipo de Doador } \\
\hline Vivo Relacionado & $9(27,3 \%)$ \\
\hline Vivo Não Relacionado & $2(6 \%)$ \\
\hline Falecido & $22(66,7 \%)$ \\
\hline
\end{tabular}

\section{- Diagnóstico da tuberculose}

O sintoma inicial mais frequente foi febre $(63,6 \%)$, seguido da perda ponderal $(21,2 \%)$ e tosse $(21,2 \%)$ (Figura 1$)$. No tocante aos sítios da TB, 24 pacientes tiveram TB localizada, sendo 13 pulmonares e 11 extra-pulmonares (peritoneal=2, ganglionar $=2$, meníngea $=2$, pleural=1, intestinal=1, renal=2, pericárdica=1). 5 tiveram formas disseminadas (pulmão + intestinal=1, pulmão+ganglionar=1, pulmão+menígea=1, pericárdica+ganglionar $=1$, pulmonar+urinária=1) e três pacientes receberam tratamento empírico, sem definição de sítio (Tabela 3). 
Sávio de Oliveira Brilhante, Silvana Daher Costa, Guilherme Aguiar Forte, Marina Santos Carvalho, Elizabeth De Francesco Daher, Tainá Veras de Sandes-Freitas, Ronaldo de Matos Esmeraldo

Tabela 2 - Tratamentos e Profilaxia pós-Tx

\begin{tabular}{lc}
\hline Imunossupresão & $\mathbf{N}=\mathbf{3 3}$ \\
\hline Indução & $17(51,5 \%)$ \\
$\quad$ Globulina Anti-timócito & $7(21,2 \%)$ \\
Anti-IL2R & \\
ISS Inicial & $24(72,7 \%)$ \\
ICN + MMA & $4(12,1 \%)$ \\
ICN + IMTOR & $5(15,2 \%)$ \\
Outros & $19(57,6 \%)$ \\
Regime com esteroide & \\
Evento por CMV & \\
Sim & $12(36,4 \%)$ \\
Rejeição Aguda do Enxerto & \\
Sim & $12(36,4 \%)$ \\
PPD pré-TX & \\
Reagente (>5mm) & $12(36,4 \%)$ \\
Não Reagente (<5mm) & $15(45,4 \%)$ \\
Não Disponível & $6(18,2 \%)$ \\
LTBI com Isoniazida & $9(27,3 \%)$ \\
\hline
\end{tabular}

Tx: transplante; Anti-IL2R: Anticorpo anti receptor de Interleucina 2; ICN: Inibidor da calcineurina; MMA: Ácido micofenólico; IMTOR: Inibidor da proteína alvo da rapamicina; CMV: Citomegalovírus; MP: Metilpredinisolona;

PPD: Proteina Purificada Derivada da tuberculina;

LTBI: tratamento de TB latente

Tabela 3 - Sítios de Tuberculose

\begin{tabular}{lc}
\hline \multicolumn{1}{c}{ Sítios } & $\mathbf{N = 3 3}$ \\
\hline Localizada & $24(72,7 \%)$ \\
Pulmonar & 13 \\
Extra-pulmonar & 11 \\
Pleural & 1 \\
Renal & 2 \\
Peritoneal & 2 \\
Ganglionar & 2 \\
Meníngea & 2 \\
Pericárdica & 1 \\
Intestinal & 1 \\
Disseminada & $5(15,2 \%)$ \\
Pulmonar + Ganglionar & 1 \\
Pulmonar + TGI & 1 \\
Pulmonar + Meníngea & 1 \\
Pericárdica + Ganglionar & 1 \\
Pulmonar + Urinária & 1 \\
Sítio não conclusivo & $4(12,1 \%)$ \\
\hline
\end{tabular}

TGI: trato gastrointestinal
Os principais testes que levaram ao diagnóstico da TB foram pesquisa de bacilo álcool-ácido resistente (BAAR) em secreções e tecidos $(n=12)$, adenosina deaminase de líquidos (ADA) $(n=4)$, biópsias $(n=6)$ e Reação em Cadeia de Polimerase $(n=1)$ (Tabela 4).

Tabela 4 - Métodos Diagnósticos

\begin{tabular}{|c|c|}
\hline Método & $\mathrm{N}=33$ \\
\hline BAAR & 12 \\
\hline ADA & 4 \\
\hline Biópsia & 6 \\
\hline PCR & 1 \\
\hline Genexpert & 1 \\
\hline Cultura & 4 \\
\hline Tratamento Empírico & 4 \\
\hline
\end{tabular}

BAAR: Bacilo Álcool-Ácido Resistente; ADA: Adenosina Deaminase; PCR: Polimerase Chain Reaction (Reação em Cadeia da Polimerase)

\section{- Tratamento da tuberculose}

Todos foram submetidos ao tratamento com rifampicina $(R)$, isoniazida $(I)$ e piridoxina $(P)$, com ou sem ethambutol (E). O tempo médio de tratamento foi de $8,4 \pm 3,8$ meses. Sete pacientes apresentaram hepatotoxicidade, dois apresentaram neuropatia periférica, três apresentaram hiperuricemia e um apresentou epigastralgia durante 0 tratamento (Tabela 5).

Tabela 5 - Esquemas terapêuticos utilizados e principais eventos adversos identificados

\begin{tabular}{lc}
\hline$\quad$ Esquema Terapêutico & $\mathbf{N}=\mathbf{3 3}$ \\
\hline RIPE & $23(69,7 \%)$ \\
RIP & $10(30,3 \%)$ \\
Tempo de tratamento (meses) & $8,4 \pm 3,8$ \\
Eventos adversos & \\
$\quad$ Hepatotoxicidade & $7(21,2 \%)$ \\
Neuropatia Periférica & $2(6,1 \%)$ \\
Hiperuricemia & $3(9,1 \%)$ \\
Epigastralgia & $1(3,0 \%)$ \\
\hline
\end{tabular}

RIPE: Rifampicina-Isoniazida-Pirazinamida-Etambutol; RIP: Rifampicina-Isoniazida-Pirazinamida

\section{- Evolução / Desfechos}

A concentração de tacrolimo (TAC) reduziu de 5,4 \pm $1,90 \mathrm{ng} / \mathrm{mL}$ pré-tratamento para $1,6 \pm 0,6 \mathrm{ng} / \mathrm{dL}$ póstratamento (menor concentração durante o tratamento com tuberculostáticos), sendo a dose aumentada em $75,5 \%$ durante o tratamento, resultando em necessidade de aumento da dose diária em mais de $70 \%$ (Figura 2). 
Considerando um período de seguimento de até 12 meses, após o fim do tratamento com tuberculostáticos, dois pacientes foram a óbito: um morreu no $4^{\circ}$ mês de tratamento por um evento vascular cerebral. $O$ segundo faleceu por infecção, 10 meses após o fim do tratamento. Um paciente perdeu o enxerto (nefropatia crônica do enxerto), o que ocorreu dois meses após o fim do tratamento.
Doze pacientes necessitaram de suspensão parcial ou completa da imunossupressão, durante o tratamento. $79 \%$ dos pacientes evoluíram com lesão renal aguda, sendo 15 pacientes KDIGO 1, oito pacientes KDIGO 2 e três pacientes KDIGO 3. Doze meses após o fim do tratamento, a função renal não retornou aos níveis basais.

Figura 1 - Manifestações clínicas iniciais da infecção por tuberculose na amostra

* Um paciente pode possuir mais de um sintoma

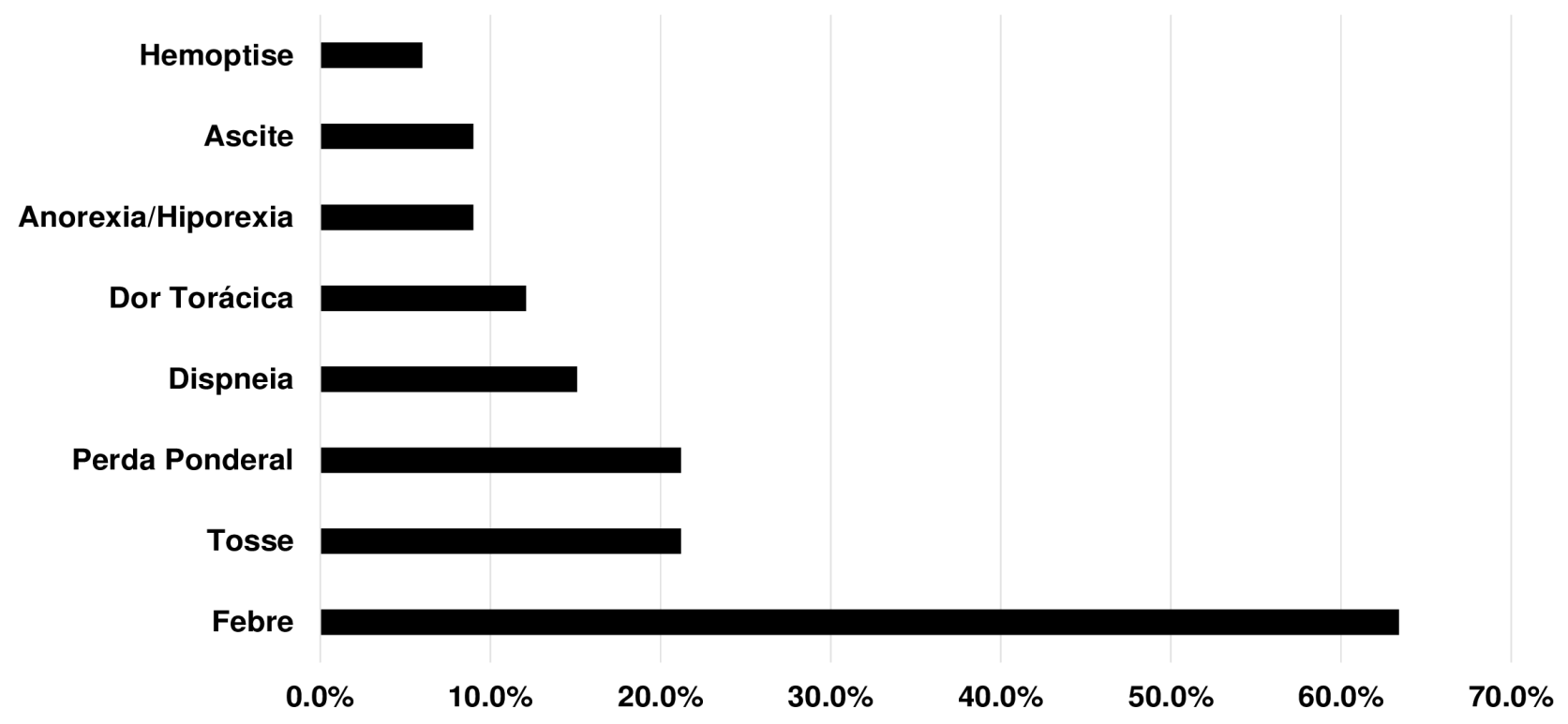

Figura 2 - Exposição ao tacrolimo (TAC) antes e durante o tratamento com tuberculostáticos

A. Dose diária de tacrolimo (TAC) (mg/dia)

18

16

14

12

10

8

6

4

2

0

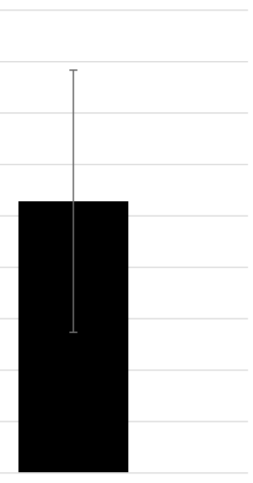

Dose Máxima de TAC durante tto
B. Concentração sanguínea de vale $(\mathrm{C} 0)$ de tacrolimo (TAC) $(\mathrm{ng} / \mathrm{mL})$

8

7

6

5

4

3

2

1

0

Nível de TAC pré-tto (CO)
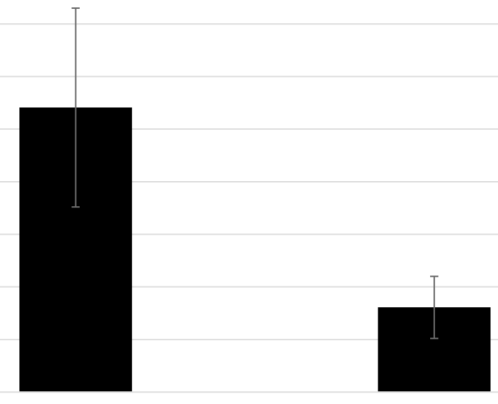

Nível Mínimo de TAC durante tto $(\mathrm{CO})$ 
Sávio de Oliveira Brilhante, Silvana Daher Costa, Guilherme Aguiar Forte, Marina Santos Carvalho, Elizabeth De Francesco Daher, Tainá Veras de Sandes-Freitas, Ronaldo de Matos Esmeraldo

\section{DISCUSSÃO}

Este estudo demonstrou que a tuberculose após o transplante é tardia, com elevado percentual de formas extra-pulmonares e está associada a desfechos desfavoráveis.

Estes resultados confirmam os achados de coortes brasileiras, que demonstraram que a tuberculose acontece tardiamente após o transplante. Há três formas de aquisição da doença TB após o transplante: transmissão através do doador, reativação e aquisição através da comunidade. $^{8}$ Apesar de pacientes imunossuprimidos terem maior risco de reativação e esta ser a principal forma de aquisição, é possível que, na nossa coorte, a realização de LTBI para pacientes de risco tenha reduzido esta forma de aquisição. ${ }^{2,9}$

Nossos resultados também são semelhantes aos da literatura quando aos sítios. Pacientes transplantados têm habitualmente elevado percentual de formas extrapulmonares.10,11 Digno de nota, em quatro pacientes, foi necessário tratamento empírico para pacientes com febre de etiologia obscura, cujos exames diagnósticos não foram conclusivos. Optamos por considerar esses casos, uma vez que o tratamento melhorou os sintomas, reforçando a hipótese diagnóstica. Essa realidade não é comum em centros americanos e europeus, mas deve ser compreendida no nosso meio. ${ }^{7}$

A existência de dois tipos de tratamento (RIP e RIPE) é consequência da modificação do tratamento realizada pelo Ministério da Saúde em 2009.12

O tratamento, frequentemente, associa-se à maior incidência de eventos adversos, como hepatotoxicidade, neuropatia periférica e interações medicamentosas com os imunossupressores, os quais foram observados e relatados no estudo. A principal interação ocorre com os inibidores da calcineurina, os quais são metabolizados pelo citocromo $\mathrm{P} 450$, que tem sua atividade estimulada pela rifampicina, reduzindo a exposição ao fármaco. ${ }^{4,8,11}$
Nossos resultados também corroboram os achados prévios de Costa e colaboradores, que demonstram que TB após o transplante está associado à lesão renal aguda e a não recuperação da função renal após o tratamento.6 Como limitação, não conseguimos identificar de forma acurada nos registros as causas de lesão renal aguda. A literatura cita como principais causas: desidratação, choque séptico, nefropatia por contraste de exames utilizados para o diagnóstico, nefrotoxicidade por fármacos utilizados em conjunto no tratamento, como antimicrobianos, nefrite intersticial aguda por fármacos e episódios de rejeição aguda, consequência da subexposição aos $\mathrm{ICN}^{3}{ }^{3}$

Apesar de nenhum paciente desta coorte ter morrido diretamente pela $\mathrm{Tb}$, dois óbitos ocorreram em até 12 meses após o tratamento, o que pode sugerir um efeito indireto da doença e seu tratamento. E, apesar de apenas um paciente ter perdido o enxerto nesse período, a função renal parece não voltar ao basal, o que foi previamente demonstrado por Costa e colaboradores. ${ }^{3}$

Nosso estudo tem limitações que devem ser apontadas. Trata-se de um estudo unicêntrico, com amostra limitada e retrospectivo. No entanto, o compartilhamento de informações sobre o diagnóstico e o manejo de infecções tropicais negligenciadas em pacientes transplantados em nosso meio é fundamental para aumentarmos o nosso acervo de conhecimento sobre esta doença.

\section{CONCLUSÃO}

Em conclusão, TB após TxR é geralmente tardia, refletindo aquisição pela comunidade; tem elevado percentual de formas extra-pulmonares e o tratamento empírico é uma realidade no nosso meio. A doença parece comprometer a sobrevida do paciente e a função do enxerto renal.

\section{AGRADECIMENTOS}

Nós somos muito gratos ao time de médicos, residentes, estudantes de Medicina e enfermeiras do Hospital Geral de Fortaleza, pela assistência provida aos pacientes e pelo suporte técnico ao desenvolvimento desta pesquisa. Esse projeto teve suporte do Conselho Nacional de Desenvolvimento Científico e Tecnológico - CNPq, através de bolsa de iniciação científica (PIBIC), do autor Sávio de Oliveira Brilhante. 


\section{ABSTRACT}

Purpose: The aim of this study was to assess demographic, clinical characteristics and outcomes of tuberculosis (TB) infection after kidney transplantation (KT). Methods: Retrospective cohort study, including KT recipients in a single transplant center located in the Northeast region of Brazil. It was included those patients whose diagnosis was performed between 2004 and $2018(n=33)$. Results: Cohort was predominantly composed by male $(54.5 \%)$, young adults $(39.8 \pm 12.3$ years-old), with median of 29.7 months after KT. Nine received treatment for latent tuberculosis infection (LTBI) due to tuberculin test $\geq 5 \mathrm{~mm}(100 \%)$. The main immunosuppressive regimen was the induction therapy with Thymoglobulin (48.4\%), and combination of tacrolimus and mycophenolate $(72.7 \%)$. The main initial symptoms were fever $(63.6 \%)$, weight loss $(21.2 \%)$ and cough $(21.2 \%)$. Twenty-four patients presented localized TB infection, 5 had disseminated infections forms, and 4 were empirically treated without defined site localization (unknown fever origin). All patients received rifampicin/isoniazid and pyrazinamide treatment, with or without ethambutol. The mean treatment time was $8.4 \pm 3.8$ months. Seven patients had hepatotoxicity and 2 presented peripheral neuropathy as treatment side-effects. The pre-treatment serum tacrolimus concentration was $5.4 \pm 1.9 \mathrm{ng} / \mathrm{mL}$, decreasing to $1.6 \pm 0.6 \mathrm{ng} /$ $\mathrm{mL}$ post treatment (lowest concentration), and a 75.5\% increase in the tacrolimus dose was required. In 12 patients, immunosuppressive regimen was partial or completely withdrawn along the TB treatment. $79 \%$ patients evolved with acute kidney injury (AKI), according to KDIGO staging, 15 patients were KDIGO 1, 8 were KDIGO 2 e 3 were KDIGO 3 , and pre-renal AKI was the main etiology. Three patients died during the course of the disease or along the period of the treatment. Conclusions: TB took place late after KT, and fever was the main initial symptom. There was a high percentage of extrapulmonary TB infections. Empirical treatment is a reality in our context. Treatment was associated to significant decrease of the calcineurin inhibitor blood concentration, probably due to the pharmacokinetic interaction with rifampicin. The percentage of renal allograft acute dysfunction was significant.

Keywords: Kidney Transplantation; Tuberculosis; Immunosuppression.

\section{REFERÊNCIAS}

1. Machado CM, Martins TC, Colturato I, Leite MS, Simione AJ, Souza MPd, et al. Epidemiology of neglected tropical diseases in transplant recipients: review of the literature and experience of a Brazilian HSCT center. Revista do Instituto de Medicina Tropical de São Paulo. 2009;51:309-24.

2. Marques IDB, Azevedo LS, Pierrotti LC, Caires RA, Sato VAH, Carmo LPF, et al. Clinical features and outcomes of tuberculosis in kidney transplant recipients in Brazil: a report of the last decade. Clinical Transplantation. 2013;27(2):E169-E76.

3. Franco-Paredes C, Jacob JT, Hidron A, RodriguezMorales AJ, Kuhar D, Caliendo AM. Transplantation and tropical infectious diseases. Int J Infect Dis. 2010 Mar;14(3):e189-196.

4. Muñoz L, Santin M. Prevention and management of tuberculosis in transplant recipients: from guidelines to clinical practice. Transplantation. 2016;100(9):1840-52.

5. ABTO. Registro Brasileiro de Transplantes: Dimensionsamento dos Transplantes no Brasil e em Cada Estado. ÓRGÃOS, A. B. D. T. D. 2017

6. Costa SD, de Sandes-Freitas TV, Jacinto CN, Martiniano LVM, Amaral YS, Paes FJVN, et al. Tuberculosis after kidney transplantation is associated with significantly impaired allograft function. Transplant Infectious Disease. 2017;19(5):e12750.
7. Viana LA. Tuberculose Diagnosticada Após O Transplante Renal. 2017.

8. Romão Junior JE. Tuberculose em pacientes transplantados renais: Desafios em países em desenvolvimento. Brazilian Journal of Nephrology. 2014;36(4):425-7.

9. De Lemos A, Vieira M, Halpern M, Quaresma R, Borchardt A, Santos M, et al. Results of implementation of preventive recommendations for tuberculosis after renal transplantation in an endemic area. American Journal of Transplantation. 2013;13(12):3230-5.

10. Subramanian AK, Morris MI, Practice tAIDCo. Mycobacterium tuberculosis Infections in Solid Organ Transplantation. American Journal of Transplantation. 2013;13(s4):68-76.

11. Meinerz G, da Silva C, Goldani J, Garcia V, Keitel E. Epidemiology of tuberculosis after kidney transplantation in a developing country. Transplant Infectious Disease. 2016;18(2):176-82.

12. Mudanças no tratamento da tuberculose. Revista de Saúde Pública. 2010;44:197-9. 